\title{
318 Correspondence
}

diagnosis of cystic fibrosis will depend on the correct interpretation of both sweat sodium and chloride in the light of the clinical findings, with support from investigation of pancreatic function.

\section{Reference}

1 Littlewood JM. The sweat test. Arch Dis Child 1986;61:1041-3.

Anne Green, Paul Griffiths, Judith Williams, and

Peter Weller

Birmingham Children's Hospital, Birmingham B16 $8 E T$

\section{Dr Littlewood comments:}

Dr Green and colleagues observe that many laboratories do not measure both sweat sodium and chloride; thus, in marginal cases, it is impossible to determine whether the chloride is higher than the sodium. While I would support the value of their observation that the chloride is commonly higher than the sodium in patients with cystic fibrosis who have marginal values and agree with their advice to measure both electrolytes, the higher chloride value in patients with cystic fibrosis can only be regarded as useful supportive evidence for the diagnosis. ${ }^{1}$

I would not accept that 'it was the interpretation of the results of the sweat test together with the natural history that excluded the diagnosis'. Unfortunately, Dr Green and colleagues provide little evidence that their small series of children with marginal results of the sweat test did not have cystic fibrosis. Certainly, normal results of a para-aminotenzoic acid test and faecal chymotrypsin test cannot be taken to exclude a minor degree of pancreatic involvement.

All evidence to the present suggests that demonstration of normal pancreatic function by pancreozymin secretin stimulation, particularly a normal bicarbonate secretion, remains the most reliable supportive evidence for the exclusion of cystic fibrosis. ${ }^{23}$

Our experience at the Leeds regional cystic fibrosis unit, derived from comprehensive assessment of over 200 patients with cystic fibrosis, in addition to many referred where the diagnosis was in doubt, suggests that individuals with cystic fibrosis who have marginal sweat electrolyte values usually have additional supportive evidence-for example, pseudomonas lung disease, obvious malabsorption, and pancreatic abnormality. Furthermore, there seems to be no direct correlation between the degree of sweat electrolyte abnormality and either the severity of the pancreatic lesion or the clinical score.

Thus I would reiterate the suggested diagnostic criteria as previously stated. ${ }^{4}$ While the relation of sodium to chloride is a valuable observation when a reliable sweat test gives a marginal result, confirmation or exclusion of the diagnosis must thereafter depend on findings other than the sweat test.

It should be re-emphasised that the common diagnostic difficulties and not uncommon actual mistakes usually result from an incorrect determination of the sweat electrolytes by the biochemist and an uncritical acceptance of the result by the clinician who also fails to show any gastrointestinai lesion or request a confirmatory sweat test at a later date.

\section{References \\ ' Henderson MJ, Littlewood JM, Miller M. Interpretation of sweat sodium and chloride concentrations. Ann Clin Biochem 1986;23:109-10. \\ 2 Smalley CA, Addy DP, Anderson CM. Does that child really have cystic fibrosis? Lancet 1979; ;:415-6. \\ 3 Wang LTK, Turtle S, Davidson AGF. Secretin pancreozymin stimulation test and confirmation of the diagnosis of cystic fibrosis. Gut 1982;23:744-50. \\ ${ }^{4}$ Littlewood JM. An overview of the management of cystic fibrosis. J R Soc Med 1986;79(Suppl 12):55-63.}

\section{Small for dates babies: are they really a problem?}

Sir,

Doctors Jones and Roberton conclude, in their recent good paper, that small for dates (SFD) babies of 37 weeks' gestation or more pose few neonatal problems. ${ }^{1}$ That this is so in their neonatal unit is because of the unit's admirable and justified reputation and of its surrounding population, in general, with a high standard of antenatal and other health care.

The only reason I write is lest the title of their paper masks an important background that has two basic features. Firstly, of infants born with low birth weight in the developing world, it is estimated the component of SFD infants over preterm infants is nearly three times greater than that in the developed world $(80 \%$ as opposed to $30 \%$ ). This suggests that a reversible condition exists: prevention of intrauterine growth failure. Under conditions in the developing world many of these SFD infants contribute to very high perinatal and infant mortality and morbidity.

Secondly, the important question of outcome. Experienced neonatologists, like these authors, know that clinically there are broadly two kinds of SFD infants: those that 'do well' and those who do not. Recent work has carried this further. ${ }^{2}$ Ultrasonography and anthropometry suggest that proportional fetal smallness, with small head size, in fetuses destined to be SFD infants leads to those who exhibit poor postnatal catch up growth, including that of head (and brain?) size. By contrast, those fetuses who are disproportionally small (with 'normal' size fetal heads) seem to exhibit good postnatal catch up. Perhaps, then, there is a distribution curve for outcome-the non-"catch uppers' at one end, with the vigorous 'catch uppers' at the other.

I suspect, because of the reasons I have suggested, that if Doctors Jones and Roberton followed their sample of SFD infants they would find them to be at the latter end.

May I repeat that my comments are the opposite of criticism of their good paper; only that their title might lead us to complacency about all SFD infants. 